45 CD11C-HI T-BET+ B CELLS CONTRIBUTE TO THE PATHOGENESIS OF SLE THROUGH GENERATION OF AUTOREACTIVE PLASMA CELLS

${ }^{1}$ Shu Wang, ${ }^{2}$ Robert W Maul, ${ }^{3}$ Varsha Kumar, ${ }^{3}$ Jingya Wang, ${ }^{4}$ Jodi L Karnell, ${ }^{5}$ Sarfaraz Hasni, ${ }^{5}$ Richard M Siegel, ${ }^{6}$ Michael P Cancro, ${ }^{3}$ Roland Kolbeck, ${ }^{2}$ Patricia J Gearhart, ${ }^{4}$ Rachel Ettinger*. ${ }^{*}$ Viela Bio, Gaithersburg MD, USA, 20878; ${ }^{2}$ National Institute on Aging, National Institutes of Health; ${ }^{3}$ Medimmune, LLC; ${ }^{4}$ Viela Bio; ${ }^{5}$ The Office of Clinical Director, National Institute of Arthritis and Musculoskeletal and Skin Diseases, National Institutes of Health; ${ }^{6}$ Department of Pathology and Laboratory Medicine, Perelman School of Medicine

\subsection{6/lupus-2019-Ism.45}

Background The etiology of systemic lupus erythematosus (SLE) is unclear, though dysregulated B cells play a key role. Several unconventional B cell subsets that associate with autoantibodies have been described in SLE. The aim of this study was to further evaluate B cell subsets in SLE and their connection to autoreactive specificities and disease manifestations.

Methods The frequency and phenotype of CD11c + Tbet $+\mathrm{B}$ cells were determined by flow cytometry. Autoantibodies were measured at the UT Southwestern core facility. The presence of $\mathrm{CD} 11 \mathrm{c}+\mathrm{B}$ cells in nephritic kidney was determined with IHC. IgH repertoires and mutational frequencies as well as transcriptome profile of $\mathrm{CD} 11 \mathrm{c}+\mathrm{B}$ cells were analyzed from RNA isolated from $\mathrm{CD} 11 \mathrm{c}+\mathrm{B}$ cells from SLE patients and compared to other $\mathrm{B}$ cell subsets from the same individual. Purified B cells from HD or SLE donors were cultured with either CD3-activated T cells or IL-21 co-stimulation and evaluated for $\mathrm{B}$ cell phenotype or production of auto-antibodies.

Results We found a CD11c hi T-bet + B cell subset highly expanded in SLE with a unique expression profile including chemokine receptors consistent with migration to target tissues. Notably, these cells were enriched for autoreactive specificities, present in nephrotic kidney and significantly correlated with specific clinical manifestations. IL-21 was a potent inducer of $\mathrm{CD} 11 \mathrm{c}$ hi $\mathrm{T}$-bet $+\mathrm{B}$ cells that further promoted the differentiation of these cells into Ig-secreting plasma cells. $\mathrm{IgH}$ repertoire analysis showed $\mathrm{CD} 11 \mathrm{c}$ hi $\mathrm{T}-\mathrm{bet}+\mathrm{B}$ cells displayed a diverse repertoire with high levels of somatic hypermutation similar to memory $\mathrm{B}$ cells and plasma cells. This diverse repertoire suggests that CD11c hi T-bet $+\mathrm{B}$ cells accumulate in response to a plethora of antigens overtime, including self-antigens in SLE.

Conclusions These results show that CD11c-hi T-bet + B cells are polyclonally expanded, and highly somatically hypermutated in SLE. Furthermore, IL-21 may be involved in their expansion and differentiation into autoreactive plasma cells, which in turn may contribute to autoimmune manifestations in SLE.

Funding Source(s): This research was supported in part by the Intramural Research Program of the NIH, National Institute on Aging and National Institute of Arthritis and Musculoskeletal and Skin Diseases and MedImmune, LLC.

\section{SMOKING EXPOSURE IN PACK-YEARS PREDICTS CUTANEOUS MANIFESTATIONS OF LUPUS}

Nnenna Ezeh*, Trevor McKown, Shivani Garg, Amanda Perez, Christie Bartels. University of Wisconsin School of Medicine and Public Health

\subsection{6/lupus-2019-Ism.46}

Background Patients of color are more likely to have systemic lupus erythematosus (SLE) and a smoking history. Prior literature notes that both smoking and race impact odds of cutaneous manifestations. Therefore, we sought to examine the impact of cumulative smoking and race on cutaneous manifestations of SLE.

Methods Our cohort study included 631 consecutive SLE patients at a single academic center. Adults with at least one ambulatory rheumatology encounter with an SLE ICD- 9 or 10 code from 2008-16 were identified. Electronic health records were manually abstracted to include patients meeting ACR 1997 or SLICC 2012 classification criteria. The primary outcomes were ACR or SLICC cutaneous criteria and SLICC Damage Index (DI) cutaneous criteria. The primary explanatory variable was smoking exposure defined as low $(<5$ packyears), medium (5-10 pack-years), and high (>10 pack-years),
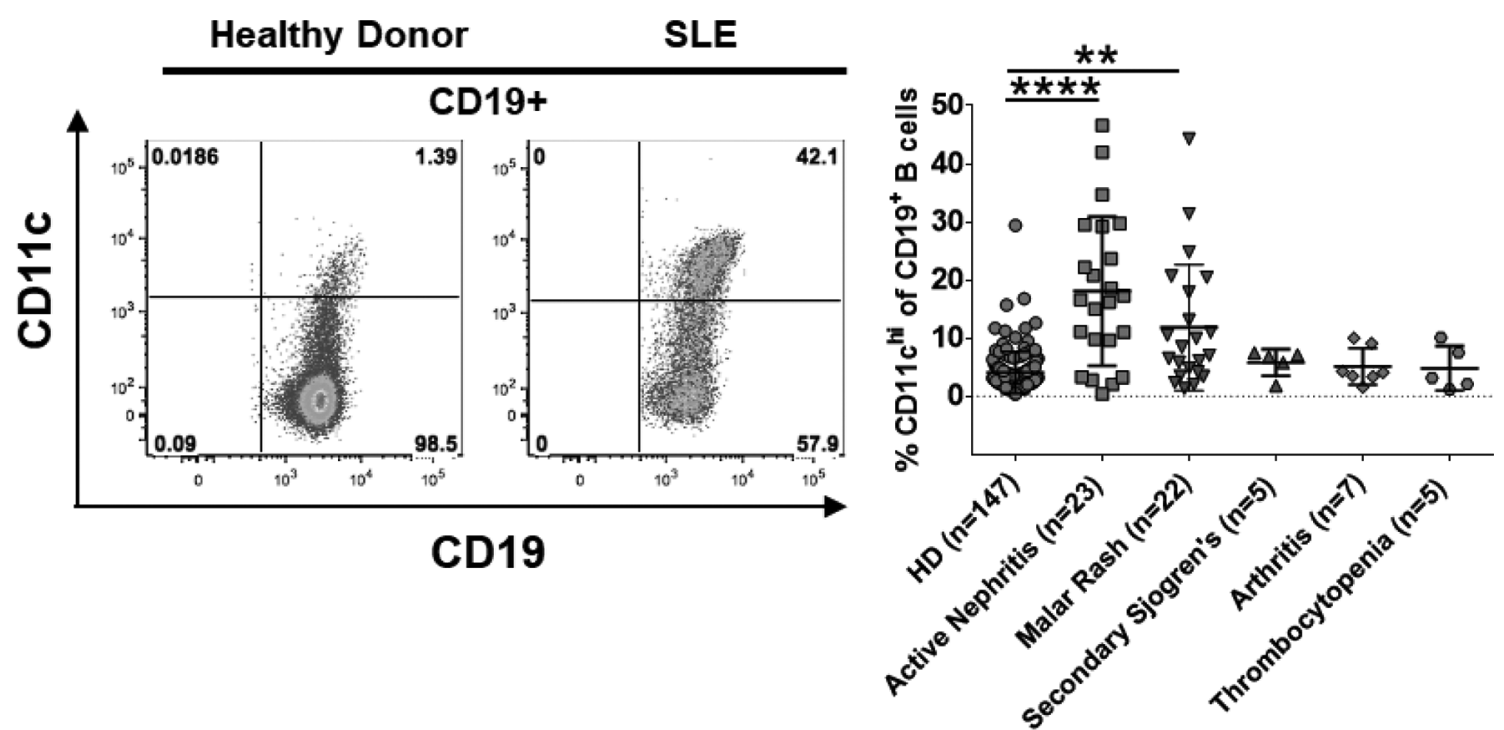

Abstract 45 Figure 1 CD11c-hi T-bet+ B cells are expanded in SLE and correlate to specific clinical manifestations. \%CD11c-hi of CD19 +B cells was determined from blood of healthy donors or SLE subjects and compared to different clinical manifestations in SLE. 
Abstract 46 Table 1 Odds ratios and $95 \% \mathrm{Cl}$ from multivariate models of cutaneous lupus by smoking exposure in pack-years

\begin{tabular}{|c|c|c|c|c|c|c|}
\hline & Acute SLICCCutaneous & Chronic SLICC Cutaneous & Any SLICC Cutaneous & Any ACR Cutaneous & Any Muco-cutaneous* & Any SLICC-DI Skin Damage \\
\hline Nonsmoker (0 pk-yrs) & ref & ref & ref & ref & ref & ref \\
\hline Low (<5 pk-yrs) & $1.6(0.8-2.9)$ & $1.7(0.8-3.9)$ & $3.7(1.3-10.6)$ & $2.0(1.0-3.8)$ & $9.0(1.2-67.7)$ & $1.8(0.6-5.7)$ \\
\hline Med (5-10 pk-yrs) & $2.3(1.1-5.1)$ & $2.0(0.8-4.9)$ & $2.1(0.8-5.8)$ & $2.0(0.9-4.3)$ & $7.3(0.95-56.2)$ & $2.6(0.8-8.3)$ \\
\hline High (>10 pk-yrs) & $1.1(0.7-1.7)$ & $2.2(1.2-4.2)$ & $1.3(0.7-2.2)$ & $1.2(0.7-1.9)$ & $0.9(0.5-1.7)$ & $4.2(1.9-9.2)$ \\
\hline Early onset & $0.6(0.3-1.3)$ & $0.9(0.3-2.7)$ & $0.6(0.2-1.7)$ & $0.7(0.3-1.6)$ & $0.6(0.2-2.2)$ & $0.8(0.2-3.7)$ \\
\hline Usual onset & ref & ref & ref & ref & ref & ref \\
\hline Late onset & $0.3(0.1-0.8)$ & $1.0(0.3-3.0)$ & $0.3(0.1-0.97)$ & $0.5(0.2-1.1)$ & $0.4(0.1-1.6)$ & $1.3(0.3-6.4)$ \\
\hline Male & ref & ref & ref & ref & ref & ref \\
\hline Female & $2.6(1.5-4.7)$ & $1.1(0.5-2.6)$ & $4.6(2.6-8.1)$ & $2.5(1.4-4.4)$ & $5.2(2.9-9.4)$ & $1.1(0.4-3.4)$ \\
\hline White & ref & ref & ref & ref & ref & ref \\
\hline Black & $0.4(0.2-0.6)$ & $1.8(0.97-3.4)$ & $0.9(0.5-1.8)$ & $0.5(0.3-0.8)$ & $0.7(0.4-1.6)$ & $2.6(1.1-5.9)$ \\
\hline Other & $0.6(0.3-1.1)$ & $3.6(1.6-8.1)$ & $0.6(0.3-1.5)$ & $0.5(0.3-1.4)$ & $0.5(0.2-1.2)$ & $2.2(0.6-8.2)$ \\
\hline
\end{tabular}

Ref= referent category; *Any Mucocutaneous included any SLICC or ACR cutaneous or mucosal criteria.

compared to nonsmokers. Covariates included age category at diagnosis (early onset $<18$ years old, 18-50 years, or late onset $>50$ years), sex, and race. Analysis was performed using multivariate logistic regression to calculate odds ratios and 95\% confidence intervals (OR, (95\% CI)).

Results Among 631 SLE patients, mean age was 42, 91\% female, $82 \%$ white, and $40 \%$ ever smokers. Patients with low smoking exposure were nine times more likely to develop any mucocutaneous manifestations (OR 9.0, (1.2, 67.7)), four times more likely to meet any SLICC cutaneous criteria (OR 3.7, (OR 1.3, 10.6)), and twice as likely to meet ACR cutaneous criteria (OR $2.0(1.0,3.8)$ ) compared to non-smokers (table 1). Patients with medium smoking exposure were twice as likely to meet acute cutaneous SLICC criteria (OR 2.3, (1.1, 5.1)), whereas those with high smoking exposure had two-fold higher odds of discoid lupus (OR 2.1, (1.1, 4.1) data not shown). Chronic cutaneous SLICC criteria and DI cutaneous criteria showed linear pack-year trends that met significance with high smoking exposure (OR 2.2, (1.2, 4.2); OR 4.2, (0.9, 9.2)). Patients of color had increased risk for alopecia, discoid lupus, chronic cutaneous lupus, and DI skin damage. Limitations included sample size and just 18\% patients of color.

Conclusions Any smoking exposure was an independent risk factor for nearly all cutaneous SLE manifestations whereas high smoking exposure and patients of color had significantly increased risk of chronic cutaneous manifestations and skin damage. Findings suggest a dose relationship between smoking exposure and cutaneous manifestations/damage, making cessation an important strategy to potentially reduce disparities and improve cutaneous outcomes in SLE.

Funding Source(s): Support: Rheumatology Research Foundation

\section{TARGETED THERAPY USING INTRADERMAL INJECTION OF ETANERCEPT FOR REMISSION INDUCTION IN DISCOID LUPUS ERYTHEMATOSUS (TARGET-DLE): RESULTS FROM A PROOF-OF-CONCEPT PHASE II TRIAL}

\footnotetext{
${ }^{1}$ Md Yuzaiful Md Yusof, 'Miriam Wittmann, 'Catherine Fernandez, 'Duncan Wilson, ${ }^{2}$ Sara Edward, 'Giuseppina Abignano, ${ }^{1}$ Adewonuola Alase, ${ }^{2}$ Philip Laws, ${ }^{1}$ Mark Goodfield, ${ }^{1}$ Paul Emery, ${ }^{1}$ Edward Vital. ${ }^{1}$ University of Leeds; ${ }^{2}$ Leeds Teaching Hospitals NHS Trust
}

Background A significant proportion of patients with discoid lupus erythematosus (DLE) are resistant to conventional therapies. Tumour necrosis factor (TNF) is pathogenic in DLE. A concern with systemic TNF-i administration is induction of pathogenic autoantibodies and flare of disease. This could be overcome using a low-dose intra-dermal injection, which may be sufficient to neutralise the TNF in lesions, without systemic TNF effects.

The objective of this trial was to assess the efficacy and safety of a novel route of administration of a TNF-i using a low dose intra-dermal injection of etanercept (ETN) for remission induction in DLE.

Methods A prospective single arm, Simons 2-stage minimax design with Hybrid adaptation, phase II open label trial was conducted in Leeds [NCT02656082]. Key inclusion criteria were i) adults aged $1880 \mathrm{y}$; ii)oneactive DLE lesion and iii) refractory to anti-malarials. One index lesion with the highest activity was treated with weekly intra-dermal injection of up to $10 \mathrm{mg}$ ETN. The primary endpoint was 6 patients achieving the modified limited Score of Activity and Damage in DLE (ML-SADDLE) 20 response (defined as reduction $20 \%$ in total activity comprises erythema, induration and scaling from baseline) at Week 12 for a Phase 3 trial to be recommended. Secondary endpoints included change in objective outcome measures; lesional thermography and laser Doppler imaging.

Results All 25 DLE patients were recruited over 18 months (18 female, mean age $47 \pm 12$ y, 6 had SLE, 9 had positive ANA and median (range) no. of previous systemic therapies was 5 (116) 17 patients completed the primary
Abstract 47 Table 1 Secondary Endpoints (per protocol; $n=17$ )

\begin{tabular}{llll}
\hline Endpoint & $\begin{array}{l}\text { Pre- } \\
\text { Treatment }\end{array}$ & $\begin{array}{l}\text { Post- } \\
\text { Treatment }\end{array}$ & p-value \\
\hline Physician VAS, mean (SD) mm & $53.1(16)$ & $23.2(20)$ & $<0.001$ \\
Patient VAS, mean (SD) mm & $56.9(28)$ & $29.7(28)$ & 0.001 \\
DLQI, mean (SD) & $11.4(7)$ & $6.5(6)$ & $<0.001$ \\
Laser Doppler Imaging, mean (SD) perfusion unit & $495.1(224)$ & $376.2(223)$ & 0.018 \\
Infrared thermography, mean (SD), ${ }^{\circ}$ Celcius & $1.92(1.17)$ & $1.08(1.05)$ & 0.005 \\
\hline
\end{tabular}

